\title{
TEORÍA FEMINISTA Y RELACIONES INTERNACIONALES: BALANCE DE CUARENTA AÑOS DE ACTIVISMO ACADÉMICO EN EL CENTENARIO DE LA DISCIPLINA
}

\author{
Leire MOURE*
}

1. Hace apenas unos meses A. J. Tickner y J. True argumentaban «que el feminismo no llegó tarde a las Relaciones Internacionales». Contrariamente fueron estas las que "llegaron tarde al feminismo» ". Esta reflexión, realizada a la luz de la conmemoración del centenario del nacimiento de la disciplina, entraña una profunda crítica a las reconstrucciones historiográficas plasmadas en lo que conocemos como la narrativa canónica de las Relaciones Internacionales ${ }^{2}$. La anterior afirmación apunta, en suma y sin ambages, al oscurecimiento y a la marginalidad intencionada que las mujeres, el género y la propia teoría feminista han padecido a lo largo del siglo xx y, en menor medida, en lo que llevamos del siglo XXI.

2. La versión revisionista del surgimiento de la disciplina apunta a que las Relaciones Internacionales «nacieron en el contexto de redes transnacionales de académicos, diplomáticos, políticos y activistas» y, contrariamente a lo mantenido tradicionalmente, "las mujeres pertenecían a esas redes y contribuyeron a la formación intelectual de la disciplina desde un enfoque genuinamente feminista, bien como miembros de grupos de presión, como la Liga Internacional de Mujeres por la Paz y la Libertad (WILPF), bien como autoras independientes o académicas». Estas pioneras pusieron de relieve

* Profesora Agregada de Derecho Internacional Público y Relaciones Internacionales, Universidad del País Vasco/Euskal Herriko Unibertsitatea (UPV/EHU) (leire.moure@ehu.eus).

1 Tickner, J. A. y True, J., «A Century of International Relations Feminism: From World War I Women's Peace Pragmatism to the Women, Peace and Security Agenda», International Studies Quarterly, vol. 62, 2018, p. 221.

2 En torno a esta cuestión desde un planteamiento crítico puede verse: ARENAL, C., Etnocentrismo y Teoría de las Relaciones Internacionales: Una visión crítica, Madrid, Tecnos, 2014. Una extensa exposición sobre la evolución en clave canónica de la disciplina se encuentra en Sodupe, K. (dir.), MourE, L., Ferrero, M. y Elizondo, I. (cols.), La teoría de las Relaciones Internacionales a comienzos del siglo XXI, Leioa, Servicio Editorial UPV/EHU, 2003. 
«los intereses de las mujeres, los niños y otros grupos marginados, exigieron representación femenina en el gobierno y la diplomacia, condenaron el imperialismo y el racismo, se opusieron al capitalismo militar, emplearon la retórica religiosa, emocional y universal, y defendieron el papel de educación» ${ }^{3}$.

3. El feminismo, en efecto, nació y evolucionó desde el activismo social mientras que las Relaciones Internacionales «tradicionales» trataban de indicar a los gobernantes las estrategias para poner fin a la guerra como fenómeno político tratando de identificar las condiciones necesarias para el establecimiento de una paz estable y duradera. Sin despreciar el papel inicial que jugaron las mujeres en aquel escenario de entreguerras, lo cierto es que las Relaciones Internacionales emergieron en un mundo de hombres, el de la alta política, la diplomacia y la guerra ${ }^{4}$ y, en un contexto académico, donde las mujeres, salvo notables excepciones, no tenían cabida ${ }^{5}$. De ahí la búsqueda de otros canales de participación en un mundo en el que la mayoría de ellas ni tan siquiera tenían derechos políticos. Es por esto que la teoría y la práctica feminista están impregnadas de un profundo escepticismo sobre todo conocimiento que pretenda ser universal y objetivo, ya que, en realidad, se basa en la vida y las experiencias de los hombres, y niega, consecuentemente, una imagen completa de la realidad ${ }^{6}$.

4. Es bien sabido que la teoría feminista no es patrimonio exclusivo de las Relaciones Internacionales y las dificultades para su afianzamiento disciplinar tampoco. El feminismo dentro de la academia constituye un saber transversal que alcanza a todas las Ciencias Sociales y las Humanidades y la incorporación a sus respectivos corpus disciplinares solo se explica por el ingreso tardío y lento de las mujeres en la educación superior desde la década de los setenta ${ }^{7}$. Desde entonces, los estudios feministas representan, sin duda, una apuesta continua y decidida por el cambio, la transformación o la emancipación social. No obstante, su impacto ha sido desigual, ha permanecido apartado de las corrientes principales y, a pesar de esta marginalidad,

3 Jan Stöckmann se refiere a autoras y activistas como Anna B. Eckstein, Agnes Headlam-Morley, Lucy Mair, Margery Perham, Helena Swanwick y Louise Weiss, en «Women, wars, and world affairs: Recovering feminist International Relations, 1915-1939», Review of International Studies, vol. 44, 2019, pp. 215-235.

4 Tickner, J. A., "Gender Research in International Relations», en SAWER, M. y BAKer, K. (eds.), Gender Innovation in Political Science: New Norms, New Knowledge, Londres, Palgrave, 2019, p. 153.

5 Esta realidad ha cambiado muy lentamente desde hace 100 años. Según datos de la ONU Mujeres en 2017 el 90 por 100 de los jefes de Estado o de gobierno, el 82 por 100 de los cargos ministeriales y el 85 por 100 de los embajadores eran hombres. Las organizaciones e instituciones internacionales también tienen un escaso historial de mujeres en cargos diplomáticos de alto nivel. Por ejemplo, las Naciones Unidas nombraron a su primera mediadora, Mary Robinson, en 2013. Por su parte, el Servicio de Acción Exterior de la Unión Europea está dirigido por Federica Mogherini, pero los nueve Enviados Especiales de la Unión son hombres. Véase Aggestam, K. y Towns, A. E., «The Study of Gender, Diplomacy and Negotiation». en Aggestam, K. y Towns, A. E. (eds.), Gendering diplomacy and international negotiation, Londres, Palgrave Macmillan, 2018, p. 2.

6 Tickner, J. A. y True, J., op. cit., nota 4, p. 228.

7 Pearsea, R., Hitchсоскв, J. N. y Keanec, H., «Gender, inter/disciplinarity and marginality in the social sciences and humanities: A comparison of six disciplines», Women's Studies International Forum, vol. 72, 2019, pp. 109-126. 
constituye hoy en día una fuente de fortaleza para las distintas áreas de conocimiento a través del cuestionamiento o «interrogatorio perpetuo» de los fundamentos ontológicos y epistemológicos de los mainstreams disciplinares $^{8}$. En resumen, las feministas han introducido las relaciones de género en la agenda, desafiando así los fundamentos del conocimiento y la producción académica que no lo tienen en cuenta.

5. En este sentido, no cabe duda que la investigación feminista ha aportado nuevas evidencias empíricas, nuevas teorías, perspectivas y debates ${ }^{9}$. Sin embargo, como veremos más adelante, estudios recientes señalan que, si bien "las ortodoxias han sido cuestionadas», en la mayoría de las ocasiones «no han logrado ser aún transformadas» ${ }^{10}$. De hecho, el reconocimiento del pensamiento feminista y la investigación de género como fuente de autoridad científica sigue poniéndose en cuestión en diferentes dominios disciplinares. Entre las disciplinas que han obtenido peores resultados en los análisis comparados realizados hasta la fecha aparecen las Relaciones Internacionales, solo superadas por el Derecho y la Economía, mientras que, en el campo de la Sociología, la Antropología o la Historia, el pensamiento feminista ha tenido un gran impacto o ha sido plenamente incorporado a sus respectivos mainstreams $^{11}$. De hecho, como bien señala J. A. Tickner «las Relaciones Internacionales han sido el campo más renuente a adoptar un enfoque de género dentro de la Ciencia Política» ${ }^{12}$.

6. Esto nos lleva a cuestionarnos sobre los motivos que han dificultado la recepción y consolidación del feminismo en nuestro ámbito de estudio. En primer lugar, como ya se ha señalado, el feminismo, desde su entrada «oficial» en las Relaciones Internacionales a finales de los ochenta del siglo pasado, ha adoptado, en términos generales, formas críticas y reflexivas de construcción de conocimiento partiendo de ontologías y epistemologías diferentes a las convencionales. Consecuentemente, ha empleado también metodologías que apuntan hacia preguntas de investigación que «abran ventanas de oportunidades para llegar a nuevas formas de conocimiento para abordar los problemas del mundo real en lugar de llenar vacíos en el conocimiento existente». En la investigación feminista, tales preguntas «deben emanar de una variedad de fuentes e informantes, incluso de la práctica, de las experiencias de las mujeres y las experiencias de individuos y organizaciones que buscan generar un cambio social» ${ }^{13}$. Por otra parte, los estudios clásicos sobre seguridad, con un carácter marcadamente masculino, han dominado la disciplina desde su nacimiento hasta fechas recientes y ello ha dificultado

\footnotetext{
8 Ibid.

9 Jenkinsa, F., Keaneb, H. y Donovanc, C., «Work (still) in progress: Establishing the value of gendered innovations in the social sciences», Women's Studies International Forum, vol. 72, 2019, pp. 104-108.

10 Pearsea, R., HitchcockB, J. N. y Keanec, H., op. cit., p. 110.

11 Ibid.

12 Tickner, J. A., op. cit., nota 4, p. 153.

13 Ackerly, B. A. y True, J., "Doing Feminist Research in Political and Social Science», Journal of International Women's Studies, vol. 5, 2010, núm. 3, p. 75.
} 
sobremanera la entrada de corrientes disidentes o de formas críticas de acercamiento a esa realidad ${ }^{14}$.

7. La observación precedente guarda relación con el hecho de que las perspectivas feministas en Relaciones Internacionales redirigen nuestra atención a cuestiones «que no han caído dentro del alcance de la disciplina o de la manera en que esta ha definido tradicionalmente el conocimiento». La visión tradicional, que dota de prioridad ontológica a los recursos materiales, a los Estados, entendidos como actores autónomos e individualistas, contrasta con el énfasis en las relaciones sociales constituidas por estructuras políticas, económicas y sociales históricamente desiguales que subraya el feminismo. Desde esta perspectiva se observa el mundo «de abajo hacia arriba», a partir de las vidas de los individuos y sus relaciones. «Trabajando de lo local a lo global», las feministas comienzan su observación desde la vida de las personas, examinando cómo están situadas en estructuras socioeconómicas cimentadas históricamente en el género y cómo estas estructuras no igualitarias impactan en sus vidas. Y así pretenden conectar a los individuos con nuevas estructuras y propuestas transformadoras de lo internacional que conduzcan a formas de gobierno global más justas e igualitarias ${ }^{15}$.

8. En suma, el feminismo entronca con el cuarto debate en la disciplina y se sitúa en el ámbito reflexivista, crítico con el mainstream disciplinar tanto desde el punto de vista ontológico como epistemológico. Ello, junto a su recepción tardía y el escaso número de mujeres internacionalistas que aún hoy conforman la academia, dificultan la incorporación, primero, y la consolidación, después, del pensamiento feminista en las Relaciones Internacionales. En este sentido, Rodríguez Manzano señalaba recientemente que si bien la teoría feminista ha "ganado cierta legitimidad en la disciplina, pues su acogida no siempre fue favorable, siendo a menudo desestimada, vista con incredulidad e, incluso con hostilidad [...] — desde aquel momento y a pesar del desarrollo experimentado- ha permanecido en sus márgenes y afrontando aún la tarea de justificar su validez intrínseca y su carácter científico» ${ }^{16}$.

9. Tras cuarenta años de pensamiento feminista en Relaciones Internacionales y pese a las resistencias y dificultades expuestas previamente, cabe señalar que en la actualidad nuestra disciplina cuenta con una extensa literatura claramente identificable ${ }^{17}$. Sin cuestionar la existencia de un nexo común, comienza a ser habitual hablar de «diversidad de aproximaciones feministas» (liberal, radical, posmoderna, poscolonial...) ${ }^{18}$ o incluso de «gene-

14 Para una explicación más extensa sobre esta cuestión, véase RodRíGuEz MANZANO, I., «En los márgenes de la disciplina: Feminismo y Relaciones Internacionales», en ARENAL, C. y SANAHUJA, J. A. (coord.), Teorías de las Relaciones Internacionales, Madrid, Tecnos, 2015, pp. 246 y 247.

15 Tickner, J. A. y True, J., op. cit., nota 7, p. 229.

16 Rodríguez Manzano, I., op. cit., nota 14, p. 267.

17 PRÜGL, E. y TICKNER, J. A., «Feminist international relations: some research agendas for a world in transition», European Journal of Politics and Gender, vol. 1, 2018, núms. 1-2, pp. 75-91.

18 Rodríguez Manzano, I., op. cit., nota 14, pp. 252-257. 
raciones» feministas ${ }^{19}$. Sin embargo, la presente reflexión no tiene por objeto realizar un recorrido en clave de categorías analíticas por las aportaciones del feminismo, ampliamente estudiadas en los trabajos ya mencionados y, sin duda, tremendamente relevantes para el avance del conocimiento en nuestro ámbito de estudio, aunque sí nos referiremos en el trayecto final de este trabajo a las agendas de investigación futuras y a los grandes ejes sobre los que debería pivotar la teoría y los estudios en esta clave.

10. Más interesante resulta, a nuestro entender, constatar a través de distintos análisis empíricos la veracidad de las críticas vertidas por el feminismo hacia la academia a lo largo de estos años. Estas se han centrado, como ya indicábamos previamente, en poner de relieve las múltiples formas en que la organización del conocimiento disciplinar puede producir y reproducir los «núcleos» de investigación que deciden cuáles son los problemas, las teorías y los métodos de un área de estudio, marginando e inhibiendo, a menudo, la producción y circulación de los enfoques feministas ${ }^{20}$.

11. Sin duda, el primer elemento que ha lastrado la consolidación del feminismo en Relaciones Internacionales es la masculinización del área. Buena cuenta de ello da el estudio TRIP (Teaching, Research and International Policy) que alberga la base de datos más completa del mundo sobre la disciplina de Relaciones Internacionales e incluye los resultados obtenidos tras encuestar a más de 5.000 profesores de Relaciones Internacionales de 32 países sobre sus tendencias de enseñanza e investigación. Desde 2004 el TRIP busca las respuestas de profesores universitarios de Relaciones Internacionales que tienen una afiliación con un departamento o institución superior de enseñanza e imparten docencia e investigan en nuestro ámbito disciplinar ${ }^{21}$. Este estudio se replicó en España a lo largo de los meses de junio y julio de $2018^{22}$. Los datos agregados del TRIP constatan que la proporción de internacionalistas mujeres se sitúa en el 33 por 100 frente a un 70 por 100 de hombres. En el caso español, el 63 por 100 de las respuestas fueron de hombres y el 37 por 100 lo fueron de mujeres. Estos porcentajes se ajustarían con bastante precisión a la población de referencia para el estudio español, 120 académicos (40

19 PrÜgl, E. y Tickner, J. A., op. cit., nota 17, p. 78.

20 Una síntesis de estas críticas pueden encontrarse en Pearsea, R., HitchcockB, J. N. y KeANec, H., op. cit., nota 7, p. 126.

21 MaliniaK, D., Peterson, S. y Tierney, M. J., TRIP Around the World: Teaching, Research, and Policy Views of International Relations Faculty in 20 Countries, 2012, https://www.wm.edu/offices/globalresearch/_documents/trip/trip_around_the_world_2011.pdf. El proyecto comenzó inicialmente en Estados Unidos liderado desde el Institute for the Theory and Practice of International Relations del College of William \& Mary en Virginia y en 2011 ya se había extendido a 20 países. Más tarde, en el informe TRIP 2014, se identificaron un total de 12.602 individuos de 32 países que cumplieron con los criterios para su inclusión en la encuesta. De estos individuos, 5.139 contestaron al cuestionario en diez idiomas diferentes, lo que elevó la tasa de respuesta por encima del 42 por 100 con un margen de error $+/-1,05$ por 100. Véase TRIP Survey, Metodology, https://trip.wm.edu/data/our-surveys/faculty-survey; WEMHEUERVogelaar, W., Bell, N., Navarrete Morales, J. M. y Tierney, M. J., «The IR of the Beholder: Examining Global IR Using the 2014 TRIP Survey», International Studies Review, vol. 18, 2016, núm. 1, p. 4.

${ }_{22}$ Moure, L., Teaching, Research, and International Policy (TRIP): Una aplicación a la Escuela Española de Relaciones Internacionales, 2019 (pendiente de publicación). 
por 100 mujeres, 60 por 100 hombres). Llama la atención el notable avance que se ha producido en este terreno. El área parece evolucionar hacia una mayor paridad entre sexos. Esto sitúa a España por encima de la media del TRIP. No obstante, si observamos los perfiles de los encuestados en nuestro país con mayor detenimiento, se puede advertir que el colectivo femenino ocupa en la mayoría de las ocasiones posiciones laborales notablemente más precarias, dato coincidente con el TRIP global ${ }^{23}$.

12. Por otra parte, España se sitúa en primer lugar entre los países que optan por el feminismo como aproximación teórica a la realidad internacional: 6,3 por 100 frente al exiguo 1,8 por 100 del TRIP total. Sin embargo, ello no debe conducirnos a un optimismo excesivo. La práctica totalidad de las contribuciones en este terreno son de mujeres. Las mismas que, como se ha señalado, ocupan en la universidad posiciones de mayor debilidad y precariedad. Por otra parte, su proyección tanto en la academia española como a nivel internacional no está en absoluto garantizada.

13. Estudios recientes señalan que los artículos escritos por mujeres son sistemáticamente menos centrales que los artículos escritos por hombres. Las mujeres tienden a ser menos citadas que los hombres. Existen, por tanto, diferencias significativas de género en los patrones de citas y ello es especialmente significativo en la promoción académica porque, como es bien sabido, la valoración del número de referencias se utilizan cada vez más como una medida clave de calidad e impacto de la investigación ${ }^{24}$.

14. Por otra parte, el TRIP también revela que, salvo en el caso español en el que se incluye a C. Enloe, ninguna feminista es incorporada entre los académicos que han tenido mayor influencia en los últimos veinte años en la disciplina. No es de extrañar si tenemos en cuenta que la «exclusión de género» en los planes de estudio en todos los niveles de enseñanza superior (grado, máster y doctorado) es una realidad palmaria. En este sentido, llama la atención el estudio realizado en la LSE que recoge el contenido de 43 programas docentes de un ciclo formativo completo en Relaciones Internacionales. La brecha de género en los materiales de enseñanza de la disciplina es abrumadora. Los autores evidencian inicialmente el principal sesgo que reproduce el patrón de exclusión femenina: «El 79,2 por 100 de los textos incluidos en la bibliografía son escritos exclusivamente por hombres, lo que no refleja

23 En Reino Unido, por ejemplo, 10 de los 11 encuestados con categoría de catedrático en la London School of Economics, centro de referencia europeo y mundial en el ámbito de las Relaciones Internacionales, fueron hombres. Véase Phull, K., Ciflikli, G. y Meibauer, G., «Gender and bias in the International Relations curriculum: Insights from reading lists», European Journal of International Relations, 2018, p. 5. Otros estudios que arrogan datos similares pueden encontrarse en: BEAULIEU, E., Boydstun, A. E., Brown, N. E., et al., «Women also know stuff: Meta-level monitoring to battle gender bias in Political Science», PS: Political Science \& Politics, vol. 50, 2017, núm. 3, pp. 779-783; KanToLA, J., "Why do all the women disappear?" Gendering processes in a Political Science department», Gender, Work and Organization, vol. 15, 2008, núm. 2, pp. 202-225.

24 MaliniaK, D., Powers, R. y Walter, B. F., «The Gender Citation Gap in International Relations», International Organization, vol. 67, 2013, pp. 889-922. Phull, K., CiflikLI, G. y MeIBauer, G., op. cit., nota 23 , p. 3. 
la representación de las mujeres ni en la disciplina profesional, ni en la disciplina publicada» ${ }^{25}$. Este sesgo se incrementa aún más en la medida en que se desciende en el nivel de estudio (grado), se introduce la variable de género (profesor), el subárea (estudios de seguridad) y la antigüedad del docente en cuestión (senior). A la luz de estos datos es evidente que persiste lo que hemos denominado una narrativa canónica de las Relaciones Internacionales en la etapa formativa más temprana. En ella los estudiantes son formados en clave mainstream o malestream. Por otra parte, las temáticas más inclusivas recaen en las asignaturas que guardan relación con las Organizaciones Internacionales o el Derecho internacional frente a los estudios de seguridad, ya mencionados, o los estudios regionales que arrojan los peores resultados. También se aprecia que los estudios de género están dominados por autoras femeninas. Estos patrones, sin duda, sugieren también un nítido estereotipo de género en los diferentes subcampos y aproximaciones teóricas.

15. La desoladora realidad que acabamos de describir, paradójicamente, se produce hoy en día en la misma universidad que dio carta de naturaleza a la teoría feminista en Relaciones Internacionales allá por 1988 con la celebración de la conferencia Women and International Relations y la posterior publicación de un número monográfico en su revista Millennium recogiendo las actas de ese encuentro.

16. En todo caso y, si bien debemos tener bien presentes las resistencias y dificultades descritas en los párrafos anteriores, el feminismo en las Relaciones Internacionales no debería renunciar a proporcionar, como señalan Prügl y Tickner, nuevas alternativas a la manera en la que se aborda tradicionalmente el conflicto, la violencia y la economía política internacional y a poner el acento en la manera en que los gobiernos, las organizaciones internacionales y los movimientos feministas intervienen para constituir y regular la realidad global ${ }^{26}$.

17. En este sentido las autoras afirman que son seis los temas urgentes que deberían abordarse para dar respuesta a los desafíos presentes. El primero se refiere a las causas constitutivas que vinculan la violencia armada y el género. En este terreno es importante desarrollar en mayor medida la investigación empírica basada en la teoría feminista interseccional y, por tanto, en el empleo de conceptos teóricos feministas y poscoloniales que tengan en cuenta la economía política. Este enfoque, según las autoras, podría conectarse con las investigaciones sobre las guerras civiles que no han logrado hasta el momento comprender la utilidad del género para el estudio de las dinámicas de violencia en estos contextos. Esto es particularmente urgente porque los esfuerzos de prevención en el marco de la lucha contra el extremismo violento pueden reforzarse si entendemos en profundidad la manera en que las mujeres son reclutadas por las distintas facciones enfrentadas en los conflictos actualmente existentes.

25 Phull, K., Ciflikli, G. y Meibauer, G., «Gender and bias in the International Relations curriculum: Insights from reading lists», op. cit., nota 23, p. 3.

26 Prügl, E. y Tickner J. A., op. cit., nota 17, p. 83. 
18. En segundo lugar, y paralelamente, el desarrollo de un programa de investigación en clave deconstructiva sigue siendo una tarea urgente para la teoría feminista en Relaciones Internacionales. La forma en que el binomio sexo/género participa en el binomio guerra/paz, el estudio de los sistemas de guerra y sus lógicas sexadas y sexuadas, las interpretaciones de fenómenos como la violencia sexual contra los hombres o la forma en que el sexo/género constituyen el combate, aportan un espacio abierto para repensar ideas comúnmente aceptadas.

19. En tercer lugar, no es posible desatender la evolución de las relaciones laborales en una economía plenamente globalizada, caracterizada por la agudización de las desigualdades, el autoritarismo sexista y las pésimas condiciones de trabajo que soportan gran parte de los migrantes. ¿Qué influencia tienen las variables género, raza, clase y ubicación geográfica para regular las relaciones laborales en las cadenas de valor transnacionales? En este terreno, la economía política global feminista ha logrado enlazar con el trabajo de geógrafas y antropólogas; sin embargo, las autoras consideran que las Relaciones Internacionales pueden aportar un conocimiento singular sobre políticas y regulaciones que trascienden las fronteras estatales, conectando, así, la política local con la política global.

20. En cuarto lugar, la teoría feminista debe prestar atención a los procesos contemporáneos de expropiación que amenazan a las poblaciones y conducen a la destrucción de nuestro planeta. ¿Cómo operan intersectorialmente el género, la raza y la clase en las prácticas contemporáneas de expropiación violenta de tierras y de destrucción ecológica? En este sentido, Prügl y Tickner señalan que la investigación feminista necesita ir más allá de la noción de acumulación marxista para dar cuenta de la violencia actual, presente en una gran variedad de procesos y prácticas económicas.

21. En quinto lugar, es necesario prestar mayor atención a la recepción del feminismo en las instituciones de gobernanza internacional ${ }^{27}$. Prügl y Tickner entienden que se deberían buscar respuesta a cómo se han absorbido las agendas feministas y cómo pueden esas agendas seguir siendo transformadoras. La contestación a esta cuestión debería responder nítidamente a las acusaciones de cooptación del feminismo por parte de las élites e instituciones neoliberales que gobiernan la economía internacional y la seguridad global. En opinión de las autoras, «el feminismo contemporáneo se enfrenta al neoliberalismo institucionalizado y al excepcionalismo antiterrorista» y, por ello, «puede haber recursos y trampas en el compromiso de los Estados y las organizaciones internacionales en este contexto histórico, y deben estudiarse en lugar de prejuzgarse» ${ }^{28}$.

22. En sexto lugar, el feminismo está en una posición única para identificar las alternativas que surgen del activismo de los movimientos sociales

27 Véase KunZ, R. y Prügl, E., «Introduction: gender experts and gender expertise», European Journal of Gender and Politics, vol. 2, 2019, núm. 1, pp. 3-21.

28 PrÜgl, E. y Tickner, J. A., op. cit., nota 17, p. 85. 
transnacionales. «¿Cómo crean los movimientos sociales y los ciudadanos alternativas a los órdenes económicos y de seguridad existentes? ¿Cómo influyen estas prácticas en el orden político y económico hegemónico? ¿Qué iniciativas ofrecen alternativas viables a las prácticas de seguridad masculinas? ¿Cómo se conectan estas prácticas con los procesos internacionales, en particular, a la luz de un renacimiento de la política de poder?».

23. Finalmente, añadiría que el activismo académico debe continuar y reforzarse más aún desde nuestros departamentos universitarios. No es suficiente tener una agenda investigadora vigorosa como la descrita si no se pone de relieve con total claridad el estado de la enseñanza de la disciplina y la estrecha conexión que existe entre las prácticas de contratación, los patrones de publicación y los programas de estudio que reflejan y perpetúan el sesgo de género en la academia. La investigación empírica en este terreno debe estar orientada a construir una disciplina, en términos de Acharya, auténticamente global ${ }^{29}$ que refleje la diversidad, sea inclusiva y represente fidedignamente las contribuciones de las mujeres y de la propia teoría feminista en las Relaciones Internacionales.

Palabras clave: teoría feminista, teoría de las Relaciones Internacionales, género.

Keywords: feminist therory, IR theory, gender.

29 AcharYA, A., «Global International Relations and Regional Worlds: A New Agenda for International Studies», International Studies Quarterly, vol. 58, 2014, núm. 4, p. 647. 